\title{
Lymphocyte activation gene-3 is associated with programmed death-ligand 1 and programmed cell death protein 1 in small cell lung cancer
}

\author{
Hui Sun ${ }^{1,2 \#}$, Jiawei Dai ${ }^{3 \#}$, Lishu Zhao ${ }^{1,2 \#}$, Jun Zhu ${ }^{1,2}$, Hao Wang ${ }^{1,2}$, Peixin Chen ${ }^{1,2}$, Hui Lu ${ }^{3}$, \\ Qiankun Chen ${ }^{4}$, Zhemin Zhang ${ }^{1,2}$
}

${ }^{1}$ Department of Medical Oncology, Shanghai Pulmonary Hospital, School of Medicine, Tongji University, Shanghai, China; ${ }^{2}$ School of Medicine, Tongji University, Shanghai, China; '3STU-Yale Joint Center for Biostatistics and Data Science, Department of Bioinformatics and Biostatistics, School of Life Sciences and Biotechnology, Shanghai Jiao Tong University, Shanghai, China; ${ }^{4}$ Department of Thoracic Surgery, Shanghai Pulmonary Hospital, Tongji University School of Medicine, Shanghai, China

Contributions: (I) Conception and design: Q Chen; Z Zhang; (II) Administrative support: Z Zhang; (III) Provision of study materials or patients: H Sun, J Dai; L Zhao; (IV) Collection and assembly of data: H Sun, J Dai; L Zhao; (V) Data analysis and interpretation: H Sun; J Dai; L Zhao; P Chen; (VI) Manuscript writing: All authors; (VII) Final approval of manuscript: All authors.

\#These authors contributed equally to this work.

Correspondence to: Zhemin Zhang. Department of Medical Oncology, Shanghai Pulmonary Hospital, Tongji University Medical School Cancer Institute, Tongji University School of Medicine, Shanghai 200433, China. Email: zhemindoc@163.com; Qiankun Chen. Department of Thoracic Surgery, Shanghai Pulmonary Hospital, Tongji University School of Medicine, Shanghai 200433, China. Email: drchen2016@126.com; Hui Lu. SJTU-Yale Joint Center for Biostatistics and Data Science, Department of Bioinformatics and Biostatistics, School of Life Sciences and Biotechnology, Shanghai Jiao Tong University, Shanghai, China. Email: huilu@sjtu.edu.cn.

Background: In recent years, immunotherapy has achieved notable success in cancer treatment. Indeed, the novel immune checkpoint lymphocyte activation gene-3 (LAG3) has shown promising therapeutic efficacy in non-small cell lung cancer. However, it is unclear about the role of LAG3 in immunotherapy and survival in small cell lung cancer (SCLC).

Methods: The expression of LAG3 in SCLC was evaluated in four public datasets. The association of LAG3 with programmed death-ligand 1 (PD-L1), programmed cell death protein 1 (PD-1), and overall survival (OS) was investigated. The LAG3-related biological processes and pathways were identified by functional analyses.

Results: LAG3 expression was detected in SCLC tumor tissues. In the cBioPortal dataset with 81 clinical SCLC samples, LAG3 expression was markedly associated with PD-1 and PD-L1 expression (both $\mathrm{P}<0.050$ ). In addition, Patients with high LAG3 expression had a trend toward a better OS $(\mathrm{P}=0.073)$. A similar survival trend was also observed in the GSE60052 dataset. Significantly, LAG3 expression was related to immunerelated biological processes, such as immune response, antigen processing and presentation, and $\mathrm{T}$ cell co-stimulation (all $\mathrm{P}<0.001$ ).

Conclusions: This study demonstrated that LAG3 is an important immune checkpoint that is closely associated with PD-1/PD-L1. LAG3 may be a promising novel immunotherapy target for SCLC.

Keywords: Lymphocyte activation gene-3 (LAG3), small cell lung cancer (SCLC), immunotherapy, programmed death-ligand 1 (PD-L1), programmed cell death protein 1 (PD-1)

Submitted Mar 22, 2021. Accepted for publication Sep 10, 2021.

doi: $10.21037 / \mathrm{atm}-21-4481$

View this article at: https://dx.doi.org/10.21037/atm-21-4481

^ ORCID: 0000-0003-4728-9107. 


\section{Introduction}

Lung cancer causes the highest morbidity and mortality amongst all malignancies worldwide $(1,2)$. Approximately $10-15 \%$ of cases can be categorized as small cell lung cancer (SCLC) which is characterized by high growth fractions and high recurrence rates, resulting in poor prognosis (3-5). Although chemotherapy is the standard first-line treatment for SCLC (6), resistance to chemotherapy hinders longterm survival. Therefore, studies exploring alternative therapeutic strategies for the treatment of patients with SCLC are urgently needed.

Some tumor cells with less immunogenicity, such as SCLC, can escape immune elimination and develop into cancers, and this can be reversed by suppressing certain immune checkpoints (7-11). Indeed, some immune checkpoint inhibitors have demonstrated notable success in treating cancers $(12,13)$. The programmed cell death protein 1/programmed death-ligand 1 (PD-1/PD-L1) inhibitors are effective in treating non-small cell lung cancer (NSCLC) (14), and when combined with first-line chemotherapy, survival in SCLC patients was significantly increased (15-18).

Unfortunately, in some cases, insensitivity to PD-1/ PD-L1 blockade can hinder its efficacy $(19,20)$, and other immune inhibitory checkpoints are now at the forefront of research, such as lymphocyte activation gene-3 (LAG3) (21). LAG3, also known as cluster of differentiation 223 (CD223), is a surface molecule first identified in 1990 (22). It is expressed on the membrane of various immunocytes, including tumorinfiltrating lymphocytes (TILs), dendritic cells (DCs), T regulatory (Treg) cells, natural killer cells, B cells, and so on $(23,24)$. As a member of the immunoglobulin superfamily, LAG3 is structurally similar to CD4, with approximately $20 \%$ homology shared at the DNA sequence (25). LAG3 shows a stronger affinity to human leukocyte antigen class II (HLA class II) expressed on antigen presenting cells (APCs) compared with CD4 and therefore inhibits the binding of HLA class II with TILs, hindering the anti-tumor response $(26,27)$. In HLA-II-positive melanoma tumors, this may facilitate immune escape with bidirectional function (24). The presence of LAG3 serves as an essential marker of T cell exhaustion, promoting T-cell apoptosis and inhibiting their proliferation and activation. Furthermore, cytokine secretion is reduced and tolerance is increased $(28,29)$. Elevated LAG3 expression has been observed on TILs of patients with various solid tumors, such as hepatocellular carcinoma and gastric carcinoma, as well as hematologic malignancies (30).

Reports have suggested that LAG3 co-functions with PD-L1 and PD-1 (12,31). In vivo experiments have shown that $\mathrm{T}$ cells may be activated if one of the pathways is blocked. The strategic blocking of both pathways resulted in an additive effect (32). Other studies have suggested that soluble LAG3 may be a potential anti-cancer vaccine (33). Thus, LAG3 may be a promising new immune checkpoint in cancer treatment. Additionally, combined inhibition of the LAG3 and PD-1 pathways may exert an additive therapeutic effect (34).

Our recent study found that similar to other types of cancers, some NSCLC patients showed LAG3-positive TILs. The expression of LAG3 could be predicted by PD-1 expression and was related to a poorer prognosis (7). However, there is a paucity of literature related to the expression of LAG3 in SCLC and how it affects survival in these patients. In this current study, four public datasets were accessed to investigate LAG3 expression in SCLC tissues (35-38). The relationship between LAG3 expression and survival, clinicopathological traits, and PD-L1 and PD-1 expression was investigated. Furthermore, functional analyses were performed to explore the LAG3-related biological processes and pathways. We present the following article in accordance with the REMARK reporting checklist (available at https:// dx.doi.org/10.21037/atm-21-4481).

\section{Methods}

\section{Acquisition of small cell lung cancer datasets}

The original RNA sequencing (RNA-seq) data and clinical characteristics of SCLC patients were obtained from the cBioPortal database (https://www.cbioportal. org/study/summary?id=sclc_ucologne_2015) and the Gene Expression Omnibus (GEO, https://www.ncbi.nlm. nih.gov/geo/) databases. The study was conducted in accordance with the Declaration of Helsinki (as revised in 2013). Datasets with less than 10 SCLC patients were excluded. Finally, 81 SCLC patients from the cBioPortal cohort (35), 79 SCLC patients from the GSE60052 cohort (36), 23 SCLC patients from the GSE43346 cohort (37), and 18 SCLC patients from the GSE149507 cohort (38) were enrolled for this study. The basic information of the enrolled datasets is summarized in Table 1. 
Table 1 Basic information of the enrolled datasets

\begin{tabular}{llccc}
\hline Datasets & Type & The number of SCLC samples & The number of NSCLC samples & The number of normal lung samples \\
\hline cBioPortal & Clinical tissues & 81 & - & - \\
GSE60052 & Clinical tissues & 79 & - & 7 \\
GSE43346 & Clinical tissues & 23 & - & - \\
GSE149507 & Clinical tissues & 18 & - & 18 \\
\hline
\end{tabular}

NSCLC, non-small cell lung cancer; SCLC, small cell lung cancer.

\section{Identification of the differentially expressed genes (DEGs)}

Based on the expression level of LAG3, the public cohort was equally divided into a high LAG3 expression group and a low LAG3 expression group. The limma $\mathrm{R}$ package was installed to search the DEGs. Following analysis, the DEGs between the high and low LAG3 expression groups were selected based on a 2 -fold change and a $\mathrm{P}$ value of 0.05 .

\section{Functional analysis}

For functional enrichment analysis, Gene Ontology (GO) $(34,39,40)$ and Kyoto Encyclopedia of Genes and Genomes (KEGG) $(41,42)$ analyses were performed. The GO results contained three parts, namely, molecular function, cellular components, and biological processes. The open-source software RStudio version 4.0.3 was used to visualize the GO and KEGG results.

\section{Statistical analysis}

Comparison of LAG3 expression between two groups was conducted using $t$-tests. Pearson correlation analysis was applied to examine the relationship between LAG3 expression and PD-L1 and PD-1 expression. Linear analysis was used to evaluate the relationship between LAG3 expression and clinicopathological traits and PDL1 and PD-1 expression. The Kaplan-Meier method was implemented to estimate survival curves, and the Cox regression model was used for correlation analysis on overall survival (OS) and clinical features, including age, gender, smoking status, staging of lung cancer, PD-1 expression, PD-L1 expression, and LAG3 expression. Variables with $\mathrm{P}<0.1$ were regarded as potential predictive markers. Statistical significance was defined as $\mathrm{P}<0.05$. All statistical tests were 2 -sided. All statistical analyses were performed with the RStudio software (version 4.0.3; https:// www.R-project.org).

\section{Results}

\section{Characterization of LAG3 expression in small cell lung cancer tissues}

LAG3 expression was detected in all SCLC tissues (Figure 1). In the GSE60052 cohort (36), the expression of LAG3 had no significant difference in 79 SCLC samples compared to normal lung tissues $(\mathrm{n}=7)$ (Figure 1A; $\mathrm{P}=0.23$ ). However, in the GSE149507 cohort (38), overexpression of LAG3 was detected in all 18 SCLC patient samples compared with normal lung tissues $(\mathrm{n}=18)$ (Figure $1 B$; $\mathrm{P}=0.0011)$.

\section{Correlation of LAG3 expression with PD-1 and PD-L1 expression}

Correlation analyses were performed on the four clinical datasets (Table 2). In the cBioPortal cohort (35), there was a significant correlation between LAG3 expression and both $\mathrm{PD}-1$ expression $(\mathrm{P}<0.001)$ and $\mathrm{PD}-\mathrm{L} 1$ expression $(\mathrm{P}=0.011)$. In the GSE60052 cohort (36), LAG3 expression was statistically associated with PD-1 expression $(\mathrm{P}=0.017)$, but no significant correlation was detected between LAG3 and PD-L1 expression $(\mathrm{P}=0.501)$. On the contrary, there was a significant correlation between LAG3 expression and PD-L1 expression in the GSE43346 cohort $(\mathrm{P}<0.001)$ (37). In the GSE149507 cohort (38), there was no statistical relationship between LAG3 expression and PD-1 expression, nor PD-L1 expression. This negative result with the GSE149507 cohort may be due to the limited sample size of SCLC patients.

Univariate and multivariate linear analyses of LAG3 expression were applied to two datasets with more than 50 SCLC patients (Tables S1,S2). In the cBioPortal cohort (35), both PD-1 ( $<<0.001)$ and PD-L1 $(\mathrm{P}=0.049)$ had a certain significance in the prediction of LAG3 expression (Table S1). In the GSE60052 cohort (36), PD-L1 was also shown to be a potential factor in predicting LAG3 expression $(\mathrm{P}=0.060$; Table S2). 

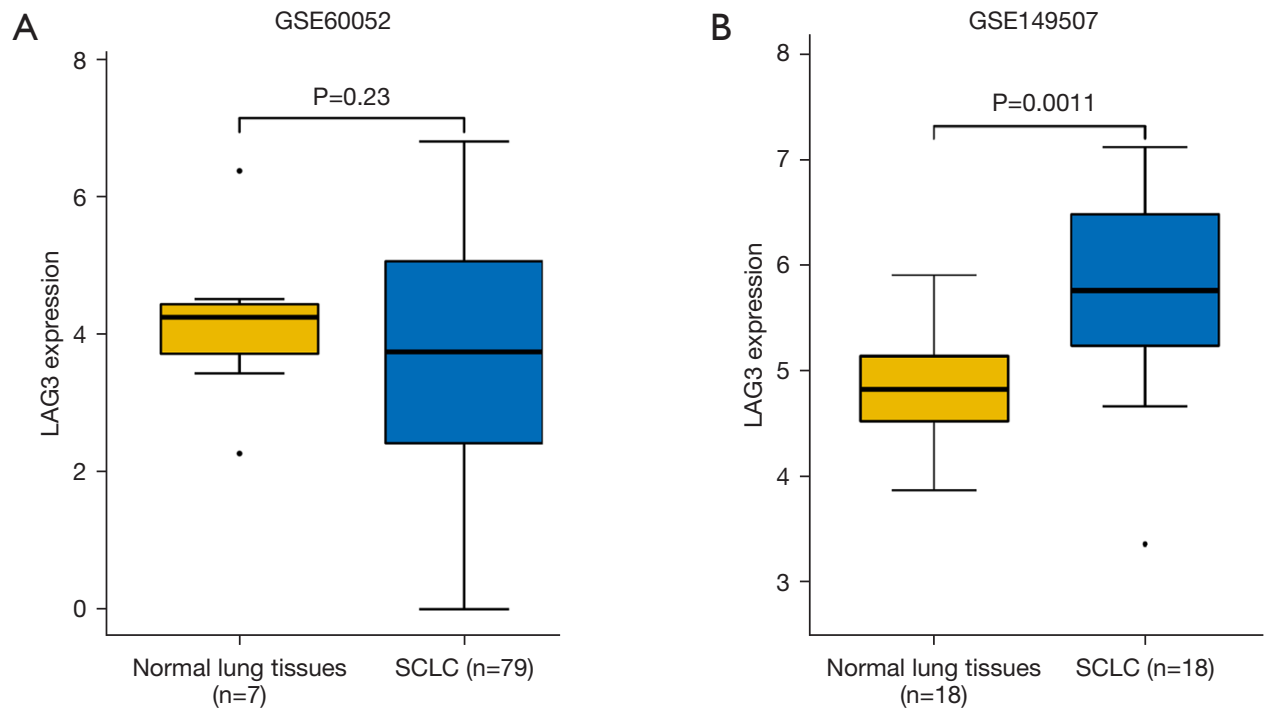

Figure 1 LAG3 expression in normal lung tissues versus SCLC tissues. (A) LAG3 expression in normal lung tissues versus SCLC tissues in the GSE60052 dataset; (B) LAG3 expression in normal lung tissues versus SCLC tissues in the GSE149507 dataset. LAG3, lymphocyte activation gene-3; SCLC, small cell lung cancer.

Table 2 Relationships between LAG3, PD-1, and PD-L1 in SCLC

\begin{tabular}{lccc}
\hline Datasets & Variables & Correlation coefficient & P value \\
\hline cBioPortal & PD-1 & 0.8599056 & 0.001 \\
& PD-L1 & 0.2800828 & 0.011 \\
GSE60052 & PD-1 & 0.267693 & 0.017 \\
GSE43346 & PD-L1 & 0.07684498 & 0.501 \\
& PD-1 & 0.2580412 & 0.235 \\
GSE149507 & PD-L1 & 0.6672631 & $<0.001$ \\
& PD-1 & -0.4078534 & 0.093 \\
\hline
\end{tabular}

Statistically significant data were marked with italics. LAG3, lymphocyte activation gene 3; PD-1, programmed cell death protein 1; PD-L1, programmed cell death protein ligand 1; SCLC, small cell lung cancer.

\section{Correlation of LAG3 expression and clinicopathologic features}

The relationship between LAG3 expression and different clinicopathologic statuses (Figures S1-S3) was examined. In three datasets with available clinical information, LAG3 expression was down-regulated in stage III-IV patients and in patients with metastasis. However, in the cBioPortal cohort (35), LAG3 was not differentially expressed between different groups (both $\mathrm{P}>0.05$ ). Similar negative results were obtained with the other two public cohorts, namely, the GSE60052 cohort (36) and the GSE149507 cohort (38). Univariate and multivariate linear analyses also suggested that clinical features failed to predict LAG3 expression (Tables S1,S2).

\section{Survival analysis}

Kaplan-Meier analysis of the cBioPortal cohort (35) revealed that patients with high LAG3 expression showed a trend toward better prognosis compared to patients with low LAG3 expression $(\mathrm{P}=0.073$; Figure $2 A)$. In the 

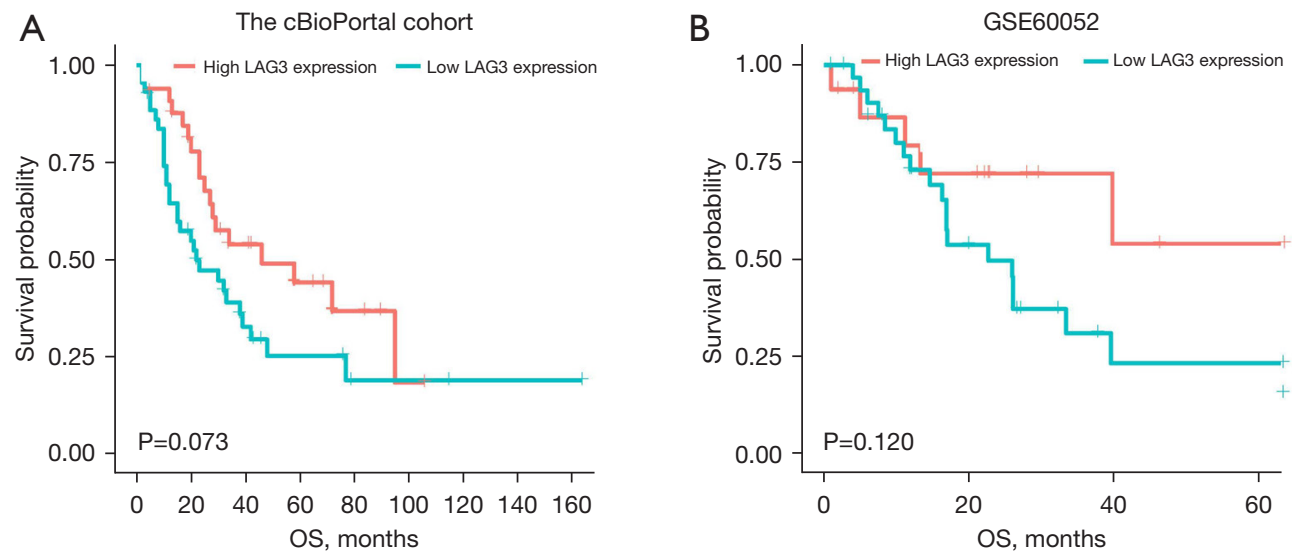

Figure 2 OS analysis in SCLC patients with different LAG3 expression levels. (A) OS analysis in SCLC patients with high versus low LAG3 expression in the cBioPortal cohort; (B) OS analysis in SCLC patients with high versus low LAG3 expression in the GSE60052 dataset. LAG3, lymphocyte activation gene-3; OS, overall survival; SCLC, small cell lung cancer.

GSE60052 cohort (36), a similar longer OS trend was observed in participants with high LAG3 expression compared to patients with low LAG3 expression $(\mathrm{P}=0.120$; Figure 2B).

\section{Cox regression analysis of $O S$}

In the cBioPortal cohort (35), gender was the only predictive factor of OS $[\mathrm{P}=0.007$; hazard ratio $(\mathrm{HR})=0.329$; 95\% confidence interval (CI): 0.142 to 0.739 ; Table 3]. Univariate cox regression analyses revealed that lung cancer staging, tumor status, lymph node status, metastasis status, and PD-1 expression were potentially significant risk factors for OS (all $\mathrm{P}<0.1$ ), but no significant association was found upon multivariate cox regression analyses (Table 3). In the GSE60052 cohort (36), low PD-1 expression was the only risk factor of $\mathrm{OS}(\mathrm{P}=0.035 ; \mathrm{HR}=2.570 ; 95 \% \mathrm{CI}: 1.071$ to 6.165; Table 4).

\section{Identification of LAG3-related signaling pathways}

The cBioPortal cohort was used to identify the LAG3related GO terms and KEGG pathways as it had the largest number of SCLC patients (35). A total of 591 DEGs were found between patients with high and low LAG3 expression. GO analysis revealed that these LAG3-related genes were linked to several immune-related processes (Figure 3 and Table 5). The top 10 LAG3-related biological processes were as follows: immune response (GO: 0006955; $\mathrm{P}<0.001)$, interferon-gamma-mediated signaling pathway
(GO: 0060333; $\mathrm{P}<0.001$ ); inflammatory response (GO: 0006954; $\mathrm{P}<0.001)$; type I interferon signaling pathway (GO: 0060337; $\mathrm{P}<0.001$ ), defense response to virus (GO: 0051607; $\mathrm{P}<0.001$ ), innate immune response (GO:0045087; $\mathrm{P}<0.001$ ), regulation of immune response (GO: 0050776; $\mathrm{P}<0.001$ ), antigen processing and presentation (GO: 0019882; $\mathrm{P}<0.001$ ), response to virus (GO: 0009615; $\mathrm{P}<0.001$ ), and $\mathrm{T}$ cell co-stimulation (GO: 0031295 ; $\mathrm{P}<0.001)$. The top $10 \mathrm{GO}$ terms related to molecular function and cellular components between the high and low LAG3 expression groups are summarized in Table 5. KEGG analysis also confirmed the close relationship between LAG3 and immunity (Figure 4 and Table 6). Staphylococcus aureus infection (hsa05150; $\mathrm{P}<0.001)$ was the most enriched KEGG pathway.

\section{Discussion}

LAG3 is a novel immune checkpoint but there is a paucity of literature related to the expression of LAG3 and its correlation with immune checkpoint PD-1/PD-L1 and patient survival in SCLC. This current study revealed the potential immunotherapeutic effects of LAG3 in patients with SCLC.

LAG3 serves as an essential marker of $\mathrm{T}$ cell exhaustion $(28,29)$. The TILs are crucial components in the anti-tumor immune response and are directly related to the development of cancer (43). The function of CD4+ and CD8+ T cells, DCs, Tregs, and so on, is regulated by inhibitory and active receptors, and can significantly impact cancer immune 
Table 3 Cox regression analysis for overall survival in the cBioPortal cohort

\begin{tabular}{|c|c|c|c|c|c|c|}
\hline Variables & \multicolumn{3}{|c|}{ Univariate } & \multicolumn{3}{|c|}{ Multivariate } \\
\hline Age (<65 vs. $\geq 65 \mathrm{y})$ & 0.955 & $0.540-1.689$ & 0.874 & - & - & - \\
\hline Sex (female vs. male) & 0.295 & $0.132-0.661$ & 0.003 & 0.329 & $0.142-0.739$ & 0.007 \\
\hline Smoking status (no vs. yes) & 0.410 & $0.056-2.996$ & 0.380 & & & \\
\hline Tumor status (T1-2 vs. T3-4) & 0.465 & $0.217-0.997$ & 0.049 & 0.671 & $0.285-1.579$ & 0.360 \\
\hline $\mathrm{N}$ status (N0 vs. N1-3) & 0.615 & $0.332-1.139$ & 0.122 & - & - & - \\
\hline Metastasis (M0 vs. M1) & 0.469 & $0.235-0.936$ & 0.032 & 0.552 & $0.247-1.233$ & 0.147 \\
\hline PD-1 expression (low vs. high) & 2.702 & $1.341-5.446$ & 0.005 & 1.958 & $0.883-4.345$ & 0.098 \\
\hline
\end{tabular}

Data with $\mathrm{P}$ value less than 0.1 were marked with italics. $\mathrm{Cl}$, confidence interval; HR, hazard ratio; LAG3, lymphocyte activation gene 3; PD-1, programmed cell death protein 1; PD-L1, programmed cell death protein ligand 1.

Table 4 Cox regression analysis for overall survival in the GSE60052 cohort

\begin{tabular}{|c|c|c|c|c|c|c|}
\hline Variables & \multicolumn{3}{|c|}{ Univariate } & \multicolumn{3}{|c|}{ Multivariate } \\
\hline Age (<65y vs. $\geq 65$ y) & 2.528 & $0.753-8.487$ & 0.134 & - & - & - \\
\hline Sex (female vs. male) & 0.993 & $0.292-3.372$ & 0.991 & - & - & - \\
\hline Smoking status (no vs. yes) & 0.647 & $0.257-1.636$ & 0.358 & - & - & - \\
\hline Tumor status (T1-2 vs. T3-4) & 0.4262 & $0.180-1.011$ & 0.053 & 0.717 & $0.294-1.746$ & 0.463 \\
\hline N status (N0 vs. N1-3) & 0.1749 & $0.049-0.630$ & 0.008 & 0.643 & $0.063-6.563$ & 0.710 \\
\hline Metastasis (M0 vs. M1) & $1,243,399$ & $0-\operatorname{Inf}$ & 0.999 & - & - & - \\
\hline PD-1 expression (low vs. high) & 2.931 & $1.276-6.731$ & 0.011 & 2.570 & $1.071-6.165$ & 0.035 \\
\hline
\end{tabular}

Data with $\mathrm{P}$ value less than 0.1 were marked with italics. $\mathrm{Cl}$, confidence interval; HR, hazard ratio; LAG3, lymphocyte activation gene 3; PD-1, programmed cell death protein 1; PD-L1, programmed cell death protein ligand 1.

escape (44). LAG3 blockade hinders the binding between LAG3 and HLA-II molecules, resulting in increased binding between HLA-II and TILs, thereby enhancing the antitumor response (45). High LAG3 expression has been observed on TILs in hematologic malignancies and various solid tumors, including hepatocellular carcinoma, gastric cancer, renal cell carcinomas, and ovarian cancer (30). In the current study, LAG3 gene expression was detected in
SCLC tissues. Additionally, previous reports using antibody or knock-down experiments showed that blocking either the PD-1 or LAG3 pathway resulted in increased activation of TILs, which led to a prolonged survival (46).

In this study, LAG3 expression was shown to be statistically correlated with PD-1 and PD-L1 expression, similar to NSCLC. Immune escape pathways are closely associated with one another (47). LAG3 is co-expressed 


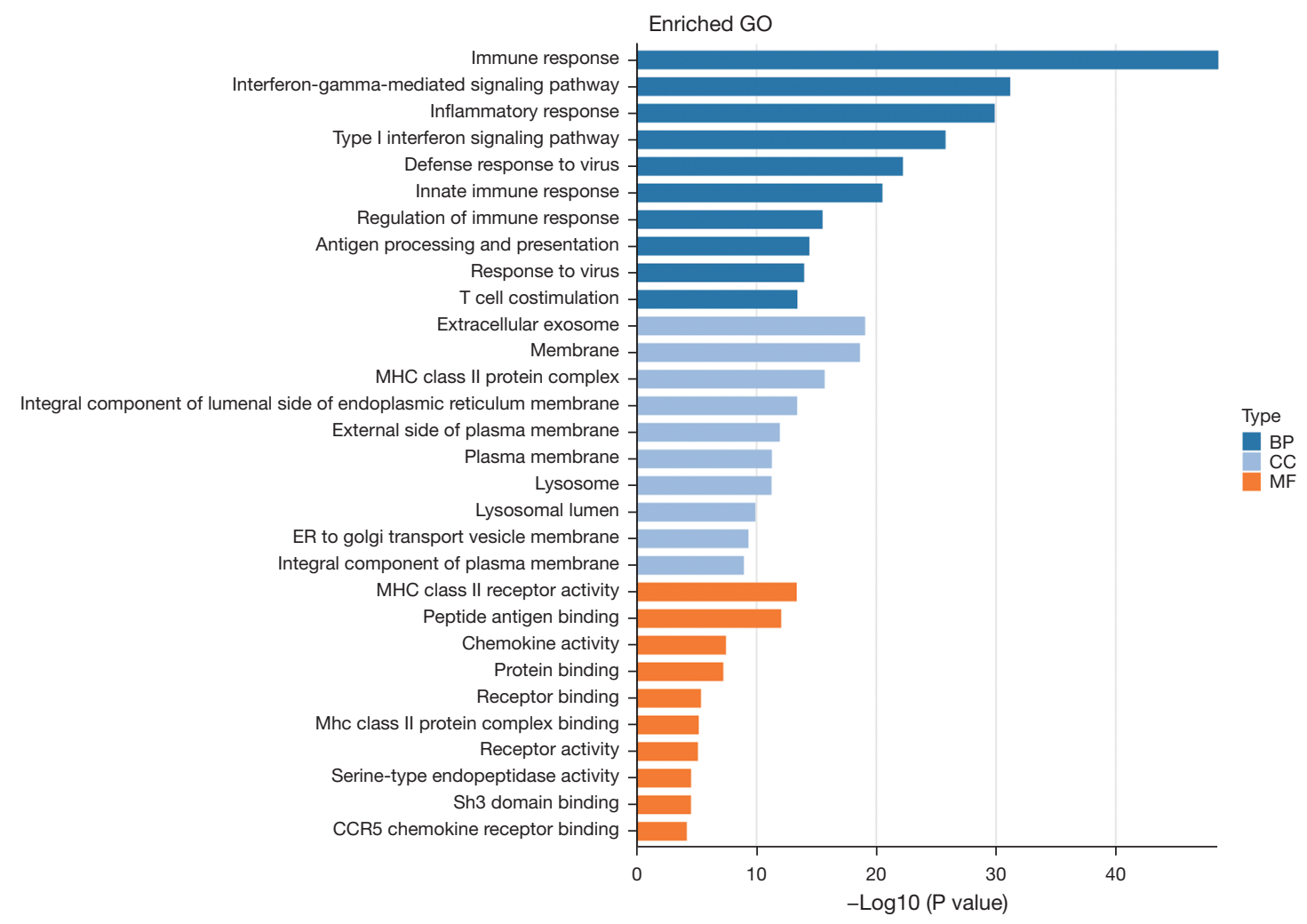

Figure 3 Enriched GO analysis of LAG3. LAG3, lymphocyte activation gene-3; GO, Gene Ontology; BP, biological process; CC, cell component; MF, molecular function.

with PD-1 on TILs and acts together to disrupt immune responses to cancer cells. This may partly explain why inhibition of the PD-1/PD-L1 pathway alone cannot lead to a notably improved prognosis in both NSCLC and SCLC. Previous researches showed that blocking both the LAG3 and PD-1 pathways resulted in superior therapeutic efficacy against cancers compared to blocking either pathway alone $(32,46,48)$. In patients who present with upregulated LAG3 expression and are insensitive to PD-1 blocking treatment, the application of this combined strategy (antiPD-1 and anti-LAG3) may improve prognosis $(30,47)$, as demonstrated in such patients with melanoma (48).

This current study demonstrated that SCLC patients with high LAG3 expression had a trend toward a better OS. In our previous researches, we performed immunohistochemical staining on tumor tissues of NSCLC and SCLC patients, and we found that NSCLC patients with LAG3-negative TILs had longer survival (7), while SCLC patients with LAG3-negative TILs had no significance in survival versus those positive (49). Given the different impacts of the abovementioned checkpoints on survival of patients with NSCLC and SCLC, the immune mechanism was considered. The immune microenvironment and immunophenotypes of SCLC appear to be distinct from that of NSCLC, which may explain the discrepancies in the ICIs efficacy and survival in these two diseases (50). Firstly, different from NSCLC over-expressing PD-L1, PD-L1 expression in SCLC was relatively low but varied greatly in the majority studies (50). It may attribute to different staining antibodies or cut-off values for positivity, biopsied tissue types, and detection platforms (51). Secondly, SCLC had a significantly lower density of TILs and higher Treg cells compared with NSCLC (52). Thirdly, HLA class IImediated antigen presentation plays a key role in activating anti-tumor immunity. However, HLA class II, the main ligand of LAG3, was rarely detected on SCLC tumor cells and HLA class II on TILs in SCLC was markedly lower than that in NSCLC (53). Therefore, all these immune factors may partly account for the different relationship between checkpoints and survival in NSCLC and SCLC and the reason for the poor efficacy of ICIs in SCLC.

In recent years, many researches have focused on 
Table 5 The top 10 GO terms of each category between the high and low LAG3 expression groups

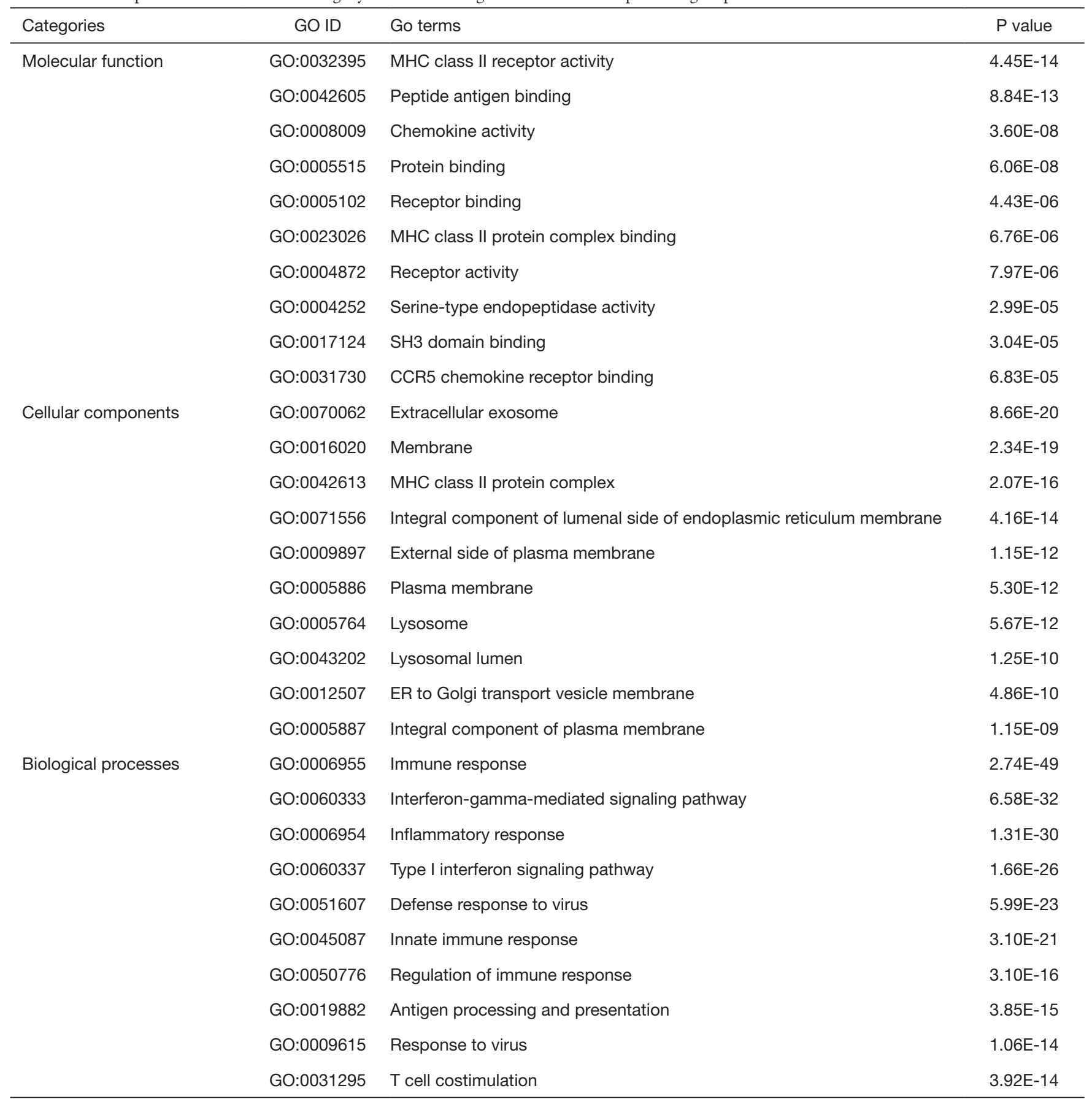

LAG3, lymphocyte activation gene 3; GO, Gene Ontology.

immunotherapy as a novel approach to achieve a favorable prognosis for patients with SCLC. LAG3 is closely associated with PD-L1 and PD-1 in expression levels and function and maybe a target to potentiate the efficacy of PD-1/PD-L1 inhibitors in SCLC. In addition, autologous TILs after stimulated by interleukin- 6 was infused into patients with anti-PD-1-resistant metastatic lung cancer and presented with general safety and clinical activity (54), which may 


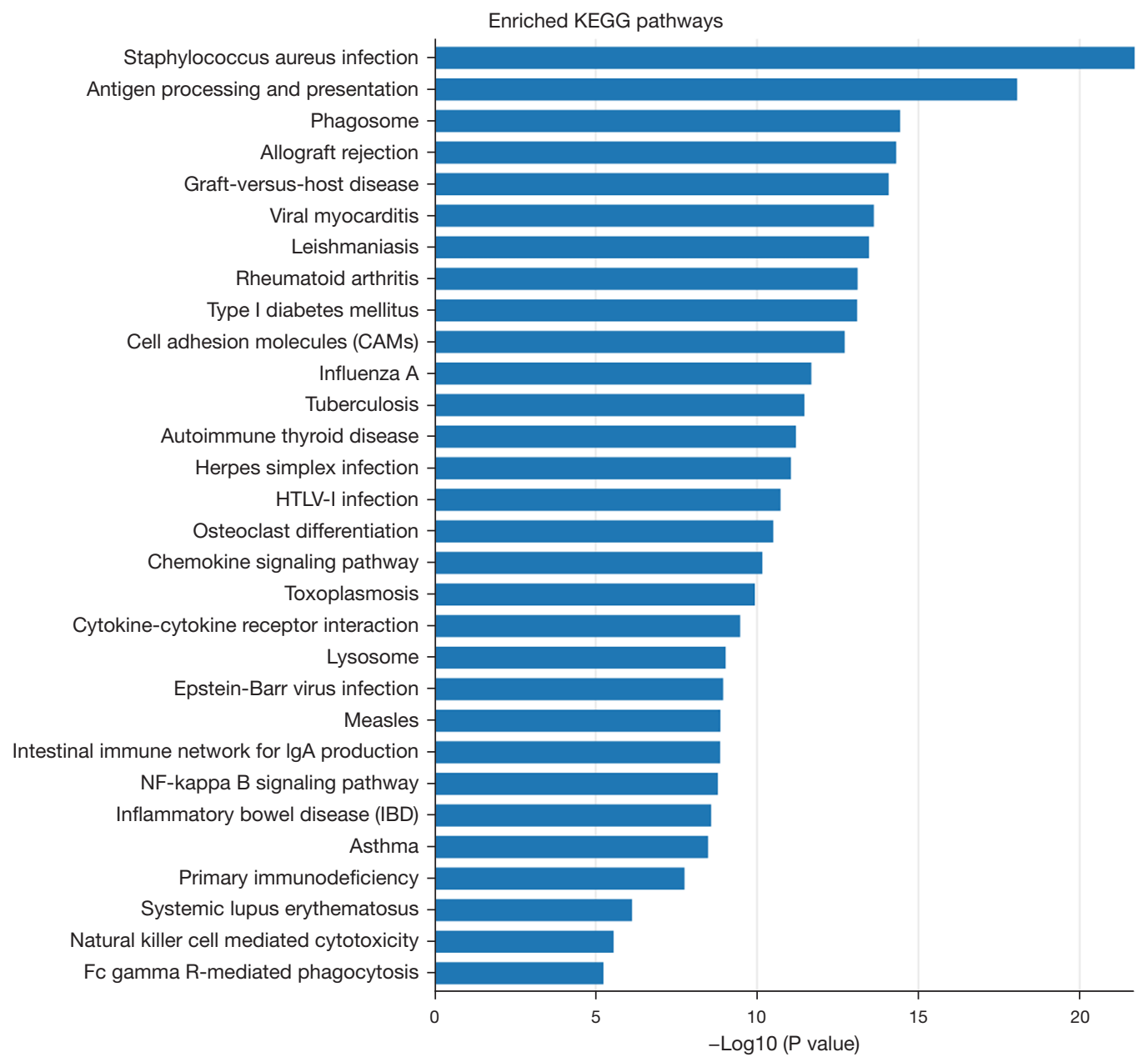

Figure 4 Enriched KEGG pathways analysis of LAG3. LAG3, lymphocyte activation gene-3; KEGG, Kyoto Encyclopedia of Genes and Genomes.

constitute a potential immunotherapy combination strategy for SCLC patients characterized by impaired antigen presentation and low-density TILs. Specifically, SCLC can be divided into four subtypes based on the dominant expression of four transcription factors: ASCL1 (SCLC-A), NeuroD1 (SCLC-N), YAP1 (SCLC-Y), and POU2F3 (SCLC-P) (55). This classification had important implications in the treatment, because SCLC-Y subtype presented with T-cell inflamed immunotype, high-expression interferon$\gamma$-associated genes, and a better prognosis, predicting the potential population that may benefit from immunotherapy or combination therapy.

There were some limitations in this investigation. First, this study was performed retrospectively. Second, some clinical data and prognostic data were not available in some datasets. Third, the sample size was small, and more data from larger populations were needed to further verify these findings.

\section{Conclusions}

In conclusion, this study revealed the correlation of LAG3 with immune checkpoint PD-1/PD-L1 and patient survival, which indicated the potential immunotherapeutic effects of LAG3 in patients with SCLC. While there has been significant progress in understanding the function of LAG3 and its interaction with other immunomarkers, its precise role in the development of SCLC remains to be fully elucidated. Furthermore, the immune responses that occur during SCLC progression and the immune checkpoints that 
Table 6 The top 10 KEGG enriched pathways of DEGs between the high and low LAG3 expression groups

\begin{tabular}{llc}
\hline KEGG ID & KEGG enriched pathways & P value \\
\hline hsa05150 & Staphylococcus aureus infection & $1.98 \mathrm{E}-22$ \\
hsa04612 & Antigen processing and presentation & $8.68 \mathrm{E}-19$ \\
hsa04145 & Phagosome & $3.66 \mathrm{E}-15$ \\
hsa05330 & Allograft rejection & $4.87 \mathrm{E}-15$ \\
hsa05332 & Graft-versus-host disease & $8.29 \mathrm{E}-15$ \\
hsa05416 & Viral myocarditis & $2.39 \mathrm{E}-14$ \\
hsa05140 & Leishmaniasis & $3.37 \mathrm{E}-14$ \\
hsa05323 & Rheumatoid arthritis & $7.62 \mathrm{E}-14$ \\
hsa04940 & Type I diabetes mellitus & $7.88 \mathrm{E}-14$ \\
hsa04514 & Cell adhesion molecules & $1.92 \mathrm{E}-13$ \\
\hline
\end{tabular}

DEGs, differentially expressed genes; LAG3, lymphocyte activation gene 3; KEGG, Kyoto Encyclopedia of Genes and Genomes.

serve as key regulators in the anti-tumor responses remain to be investigated.

\section{Acknowledgments}

Funding: This study was supported by grants from the National Natural Science Foundation of China (81802255), the Clinical Research Project of Shanghai Pulmonary Hospital (FKLY20010 and FK18005), the Young Talents in Shanghai (2019 QNBJ), the 'Dream Tutor' Outstanding Young Talents Program (fkyq1901), the Key Discipline in 2019 (Oncology), the Project of Shanghai Municipal Science and Technology Commission, the Scientific Research Project of Shanghai Pulmonary Hospital (fkcx1903), the Shanghai Municipal Commission of Health and Family Planning (2017YQ050), the Innovation Training Project of SITP of Tongji University, the Key Projects of Leading Talent (19411950300), the Youth Project of Hospital Management Research Fund of Shanghai Hospital Association (Q1902037), National Key R\&D Program of China (2018YFC0910500), and the Neil Shen's SJTU Medical Research Fund.

\section{Footnote}

Reporting Checklist: The authors have completed the REMARK reporting checklist. Available at https://dx.doi. org/10.21037/atm-21-4481

Conflicts of Interest: All authors have completed the ICMJE uniform disclosure form (available at https://dx.doi. org/10.21037/atm-21-4481). The authors have no conflicts of interest to declare.

Ethical Statement: The authors are accountable for all aspects of the work in ensuring that questions related to the accuracy or integrity of any part of the work are appropriately investigated and resolved. The study was conducted in accordance with the Declaration of Helsinki (as revised in 2013).

Open Access Statement: This is an Open Access article distributed in accordance with the Creative Commons Attribution-NonCommercial-NoDerivs 4.0 International License (CC BY-NC-ND 4.0), which permits the noncommercial replication and distribution of the article with the strict proviso that no changes or edits are made and the original work is properly cited (including links to both the formal publication through the relevant DOI and the license). See: https://creativecommons.org/licenses/by-nc-nd/4.0/.

\section{References}

1. Sung H, Ferlay J, Siegel RL, et al. Global Cancer Statistics 2020: GLOBOCAN Estimates of Incidence and Mortality Worldwide for 36 Cancers in 185 Countries. CA Cancer J Clin 2021;71:209-49.

2. Global Burden of Disease Cancer Collaboration; Fitzmaurice C, Abate D, et al. Global, Regional, and National Cancer Incidence, Mortality, Years of Life Lost, Years Lived With Disability, and Disability-Adjusted LifeYears for 29 Cancer Groups, 1990 to 2017: A Systematic Analysis for the Global Burden of Disease Study. JAMA Oncol 2019;5:1749-68.

3. van Meerbeeck JP, Fennell DA, De Ruysscher DK. Smallcell lung cancer. Lancet 2011;378:1741-55.

4. Travis WD. Advances in neuroendocrine lung tumors. Ann Oncol 2010;21 Suppl 7:vii65-71.

5. Zakowski MF. Pathology of small cell carcinoma of the lung. Semin Oncol 2003;30:3-8.

6. Rudin CM, Giaccone G, Ismaila N. Treatment of SmallCell Lung Cancer: American Society of Clinical Oncology Endorsement of the American College of Chest Physicians Guideline. J Oncol Pract 2016;12:83-6.

7. $\mathrm{He} \mathrm{Y,} \mathrm{Yu} \mathrm{H,} \mathrm{Rozeboom} \mathrm{L,} \mathrm{et} \mathrm{al.} \mathrm{LAG-3} \mathrm{Protein}$ 
Expression in Non-Small Cell Lung Cancer and Its Relationship with PD-1/PD-L1 and Tumor-Infiltrating Lymphocytes. J Thorac Oncol 2017;12:814-23.

8. He Y, Rozeboom L, Rivard CJ, et al. MHC class II expression in lung cancer. Lung Cancer 2017;112:75-80.

9. Jia K, He Y, Dziadziuszko R, et al. T cell immunoglobulin and mucin-domain containing-3 in non-small cell lung cancer. Transl Lung Cancer Res 2019;8:895-906.

10. Chen P, Zhang L, Zhang W, et al. Galectin-9-based immune risk score model helps to predict relapse in stage I-III small cell lung cancer. J Immunother Cancer 2020;8:e001391.

11. Dunn GP, Old LJ, Schreiber RD. The immunobiology of cancer immunosurveillance and immunoediting. Immunity 2004;21:137-48.

12. Woo SR, Turnis ME, Goldberg MV, et al. Immune inhibitory molecules LAG-3 and PD-1 synergistically regulate $\mathrm{T}$-cell function to promote tumoral immune escape. Cancer Res 2012;72:917-27.

13. Takaya S, Saito H, Ikeguchi M. Upregulation of Immune Checkpoint Molecules, PD-1 and LAG-3, on CD4+ and CD8+ T Cells after Gastric Cancer Surgery. Yonago Acta Med 2015;58:39-44.

14. Reck M, Rodríguez-Abreu D, Robinson AG, et al. Five-Year Outcomes With Pembrolizumab Versus Chemotherapy for Metastatic Non-Small-Cell Lung Cancer With PD-L1 Tumor Proportion Score $\geq 50$. J Clin Oncol 2021;39:2339-49.

15. Guo H, He Y, Chen P, et al. Combinational immunotherapy based on immune checkpoints inhibitors in small cell lung cancer: is this the beginning to reverse the refractory situation? J Thorac Dis 2020;12:6070-89.

16. Brahmer JR, Tykodi SS, Chow LQ, et al. Safety and activity of anti-PD-L1 antibody in patients with advanced cancer. N Engl J Med 2012;366:2455-65.

17. Horn L, Mansfield AS, Szczęsna A, et al. First-Line Atezolizumab plus Chemotherapy in Extensive-Stage Small-Cell Lung Cancer. N Engl J Med 2018;379:2220-9.

18. Ragavan M, Das M. Systemic Therapy of Extensive Stage Small Cell Lung Cancer in the Era of Immunotherapy. Curr Treat Options Oncol 2020;21:64.

19. Zhao X, Subramanian S. Intrinsic Resistance of Solid Tumors to Immune Checkpoint Blockade Therapy. Cancer Res 2017;77:817-22.

20. Shi H, Lan J, Yang J. Mechanisms of Resistance to Checkpoint Blockade Therapy. Adv Exp Med Biol 2020;1248:83-117.

21. He Y, Rivard CJ, Rozeboom L, et al. Lymphocyte- activation gene-3, an important immune checkpoint in cancer. Cancer Sci 2016;107:1193-7.

22. Triebel F, Jitsukawa S, Baixeras E, et al. LAG-3, a novel lymphocyte activation gene closely related to CD4. J Exp Med 1990;171:1393-405.

23. Lichtenegger FS, Rothe M, Schnorfeil FM, et al. Targeting LAG-3 and PD-1 to Enhance T Cell Activation by Antigen-Presenting Cells. Front Immunol 2018;9:385.

24. Chen P, Guo H, Liu Y, et al. Aberrant methylation modifications reflect specific drug responses in small cell lung cancer. Genomics 2021;113:1114-26.

25. He Y, Wang Y, Zhao S, et al. sLAG-3 in non-smallcell lung cancer patients' serum. Onco Targets Ther 2018;11:4781-4.

26. Baixeras E, Huard B, Miossec C, et al. Characterization of the lymphocyte activation gene 3 -encoded protein. A new ligand for human leukocyte antigen class II antigens. J Exp Med 1992;176:327-37.

27. Huard B, Prigent P, Tournier M, et al. CD4/major histocompatibility complex class II interaction analyzed with CD4- and lymphocyte activation gene-3 (LAG-3)-Ig fusion proteins. Eur J Immunol 1995;25:2718-21.

28. Liu Y, Chen P, Wang H, et al. The landscape of immune checkpoints expression in non-small cell lung cancer: a narrative review. Transl Lung Cancer Res 2021;10:1029-38.

29. Nguyen LT, Ohashi PS. Clinical blockade of PD1 and LAG3--potential mechanisms of action. Nat Rev Immunol 2015;15:45-56.

30. Long L, Zhang X, Chen F, et al. The promising immune checkpoint LAG-3: from tumor microenvironment to cancer immunotherapy. Genes Cancer 2018;9:176-89.

31. Liu Q, Qi Y, Zhai J, et al. Molecular and Clinical Characterization of LAG3 in Breast Cancer Through 2994 Samples. Front Immunol 2021;12:599207.

32. Blackburn SD, Shin H, Haining WN, et al. Coregulation of CD8+ T cell exhaustion by multiple inhibitory receptors during chronic viral infection. Nat Immunol 2009;10:29-37.

33. Fougeray S, Brignone C, Triebel F. A soluble LAG3 protein as an immunopotentiator for therapeutic vaccines: Preclinical evaluation of IMP321. Vaccine 2006;24:5426-33.

34. Mi H, Muruganujan A, Ebert D, et al. PANTHER version 14: more genomes, a new PANTHER GO-slim and improvements in enrichment analysis tools. Nucleic Acids Res 2019;47:D419-26.

35. George J, Lim JS, Jang SJ, et al. Comprehensive genomic 
profiles of small cell lung cancer. Nature 2015;524:47-53.

36. Jiang L, Huang J, Higgs BW, et al. Genomic Landscape Survey Identifies SRSF1 as a Key Oncodriver in Small Cell Lung Cancer. PLoS Genet 2016;12:e1005895.

37. Sato T, Kaneda A, Tsuji S, et al. PRC2 overexpression and PRC2-target gene repression relating to poorer prognosis in small cell lung cancer. Sci Rep 2013;3:1911.

38. Cai L, Liu H, Huang F, et al. Cell-autonomous immune gene expression is repressed in pulmonary neuroendocrine cells and small cell lung cancer. Commun Biol 2021;4:314.

39. Ashburner M, Ball CA, Blake JA, et al. Gene ontology: tool for the unification of biology. The Gene Ontology Consortium. Nat Genet 2000;25:25-9.

40. Gene Ontology Consortium. The Gene Ontology resource: enriching a GOld mine. Nucleic Acids Res 2021;49:D325-34.

41. Ogata H, Goto S, Sato K, et al. KEGG: Kyoto Encyclopedia of Genes and Genomes. Nucleic Acids Res 1999;27:29-34.

42. Kanehisa M, Furumichi M, Sato Y, et al. KEGG: integrating viruses and cellular organisms. Nucleic Acids Res 2021;49:D545-51.

43. Santoiemma PP, Powell DJ Jr. Tumor infiltrating lymphocytes in ovarian cancer. Cancer Biol Ther 2015;16:807-20.

44. Xu Y, Wang L, Li W, et al. Killer immunoglobulinlike receptors/human leukocyte antigen class-I, a crucial immune pathway in cancer. Ann Transl Med 2020;8:244.

45. Andrews LP, Marciscano AE, Drake CG, et al. LAG3 (CD223) as a cancer immunotherapy target. Immunol Rev 2017;276:80-96.

46. Harris-Bookman S, Mathios D, Martin AM, et al. Expression of LAG-3 and efficacy of combination treatment with anti-LAG-3 and anti-PD-1 monoclonal antibodies in glioblastoma. Int J Cancer 2018;143:3201-8.
47. Datar I, Sanmamed MF, Wang J, et al. Expression Analysis and Significance of PD-1, LAG-3, and TIM-3 in Human Non-Small Cell Lung Cancer Using Spatially Resolved and Multiparametric Single-Cell Analysis. Clin Cancer Res 2019;25:4663-73.

48. Lipson EJ, Tawbi HAH, Schadendorf K, et al. Relatlimab (RELA) plus nivolumab (NIVO) versus NIVO in firstline advanced melanoma: Primary phase III results from RELATIVITY-047 (CA224-047). 2021 ASCO Annual Meeting. Abstract 9503. Presented June 6, 2021.

49. Jiang $M$, Wu C, Zhang L, et al. FOXP3-based immune risk model for recurrence prediction in small-cell lung cancer at stages I-III. J Immunother Cancer 2021;9:e002339.

50. Sabari JK, Lok BH, Laird JH, et al. Unravelling the biology of SCLC: implications for therapy. Nat Rev Clin Oncol 2017;14:549-61.

51. Tian Y, Zhai X, Han A, et al. Potential immune escape mechanisms underlying the distinct clinical outcome of immune checkpoint blockades in small cell lung cancer. J Hematol Oncol 2019;12:67.

52. Remon J, Aldea M, Besse B, et al. Small cell lung cancer: a slightly less orphan disease after immunotherapy. Ann Oncol 2021;32:698-709.

53. Chen $\mathrm{P}$, Zhao L, Wang H, et al. Human leukocyte antigen class II-based immune risk model for recurrence evaluation in stage I-III small cell lung cancer. J Immunother Cancer 2021;9:e002554.

54. Creelan BC, Wang C, Teer JK, et al. Tumor-infiltrating lymphocyte treatment for anti-PD-1-resistant metastatic lung cancer: a phase 1 trial. Nat Med 2021;27:1410-8.

55. Owonikoko TK, Dwivedi B, Chen Z, et al. YAP1 Expression in SCLC Defines a Distinct Subtype With T-cell-Inflamed Phenotype. J Thorac Oncol 2021;16:464-76.

(English Language Editor: J. Jones)
Cite this article as: Sun H, Dai J, Zhao L, Zhu J, Wang H, Chen P, Lu H, Chen Q, Zhang Z. Lymphocyte activation gene- 3 is associated with programmed death-ligand 1 and programmed cell death protein 1 in small cell lung cancer. Ann Transl Med 2021;9(18):1468. doi: 10.21037/atm-21-4481 


\section{Supplementary}
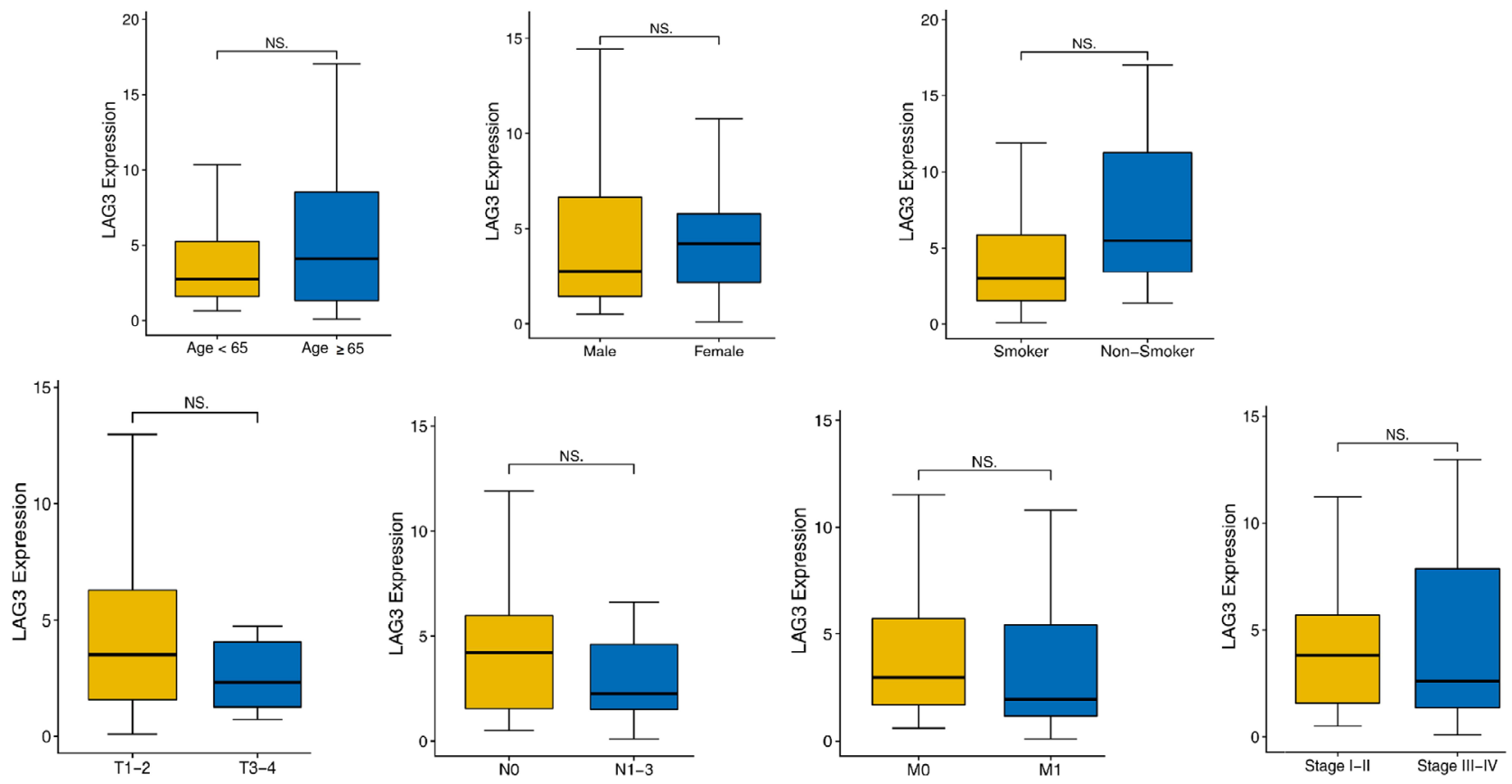

Figure S1 Correlation between LAG3 and clinical data in the cBioportal cohort. NS means no statistical significance. 

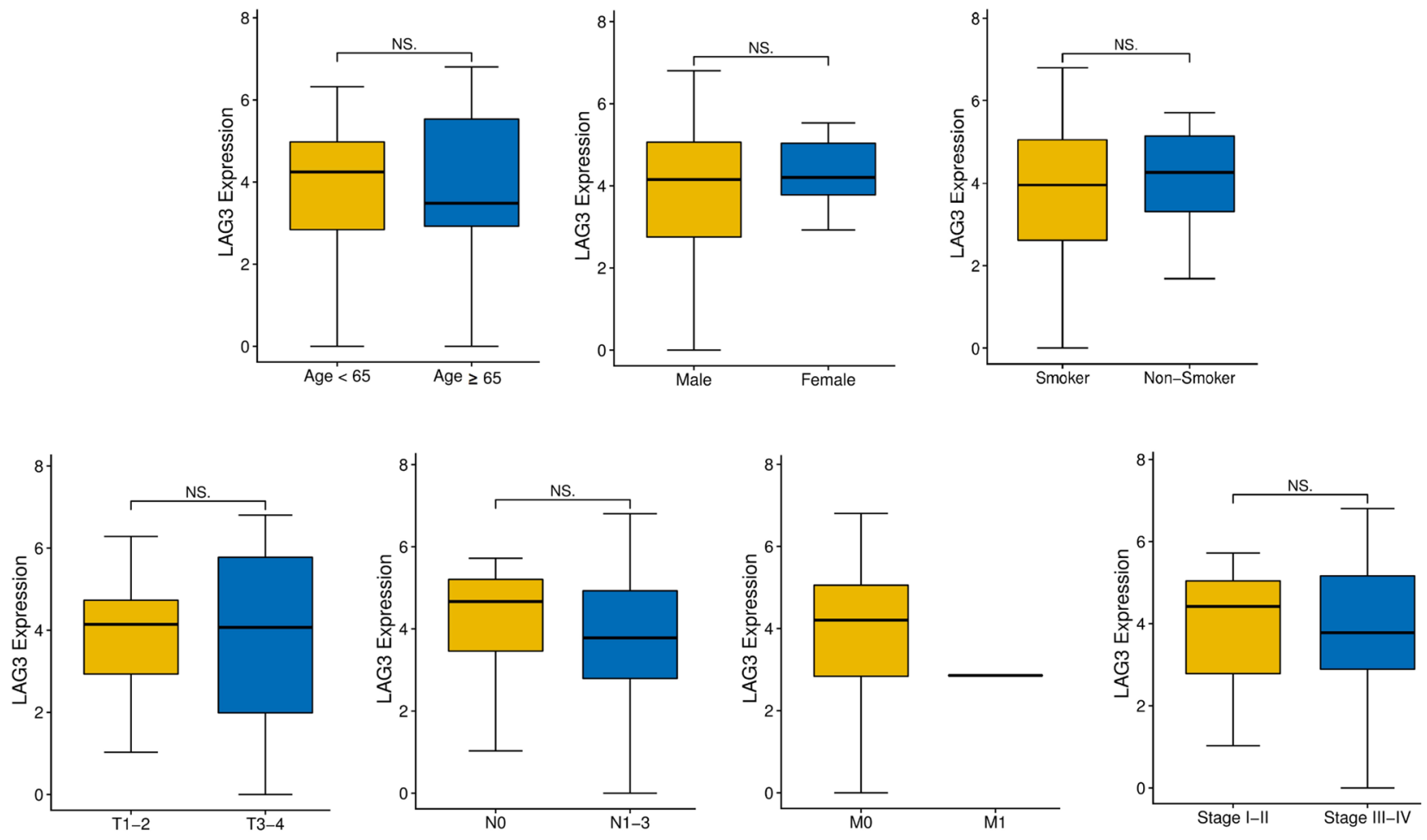

Figure S2 Correlation between LAG3 and clinical data in the GSE60052 cohort. NS means no statistical significance. LAG3, lymphocyte activation gene-3. 

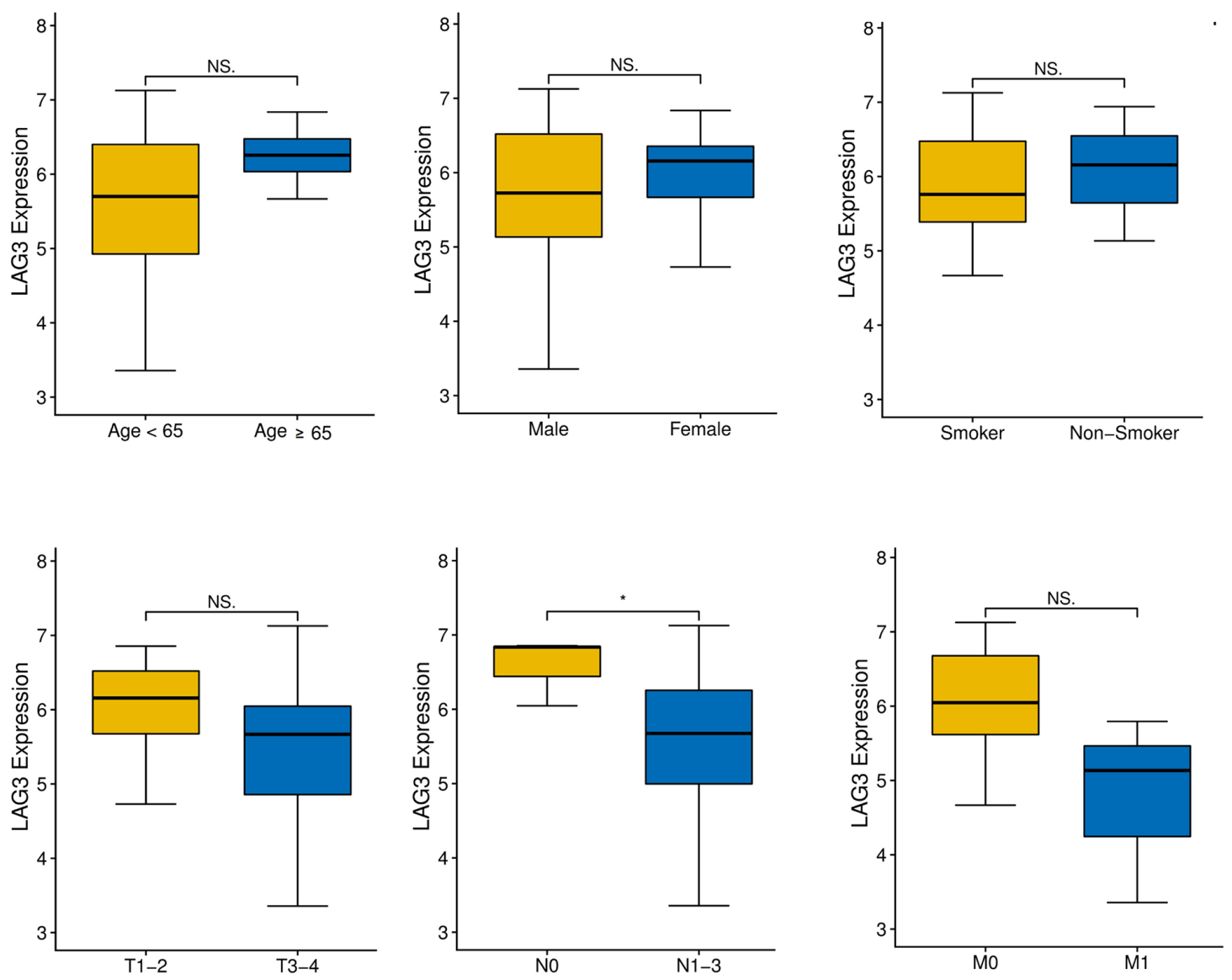

Figure S3 Correlation between LAG3 and clinical data in the GSE149507 cohort. “*” means $\mathrm{P}<0.05$; NS means no statistical significance. 
Table S1 Liner analysis for LAG3 expression in the cBioPortal cohort

\begin{tabular}{|c|c|c|c|c|c|}
\hline Variables & \multicolumn{3}{|c|}{ Univariate } & \multicolumn{2}{|c|}{ Multivariate } \\
\hline Age (<65 vs. $\geq 65$ y) & -1.0914 & 0.01884 & 0.222 & & \\
\hline Sex (female vs. male) & 0.3660 & 0.001769 & 0.709 & & \\
\hline Smoking status (non-smoker vs. smoker) & 3.5942 & 0.0303 & 0.127 & & \\
\hline Tumor status (T1-2 vs. T3-4) & -0.3991 & 0.008815 & 0.436 & & \\
\hline $\mathrm{N}$ status (N0 vs. N1-3) & -0.3299 & 0.006899 & 0.491 & & \\
\hline Metastasis (M0 vs. M1) & -0.7885 & 0.007022 & 0.484 & & \\
\hline PD-1 expression & 2.0994 & 0.7394 & $<0.001$ & 2.04406 & $<0.001$ \\
\hline
\end{tabular}

Data with $\mathrm{P}$ value less than 0.1 were marked with italics. LAG3, lymphocyte activation gene 3; PD-1, programmed death 1; PD-L1, programmed death ligand 1.

Table S2 Liner analysis for LAG3 expression in the GSE60052 cohort

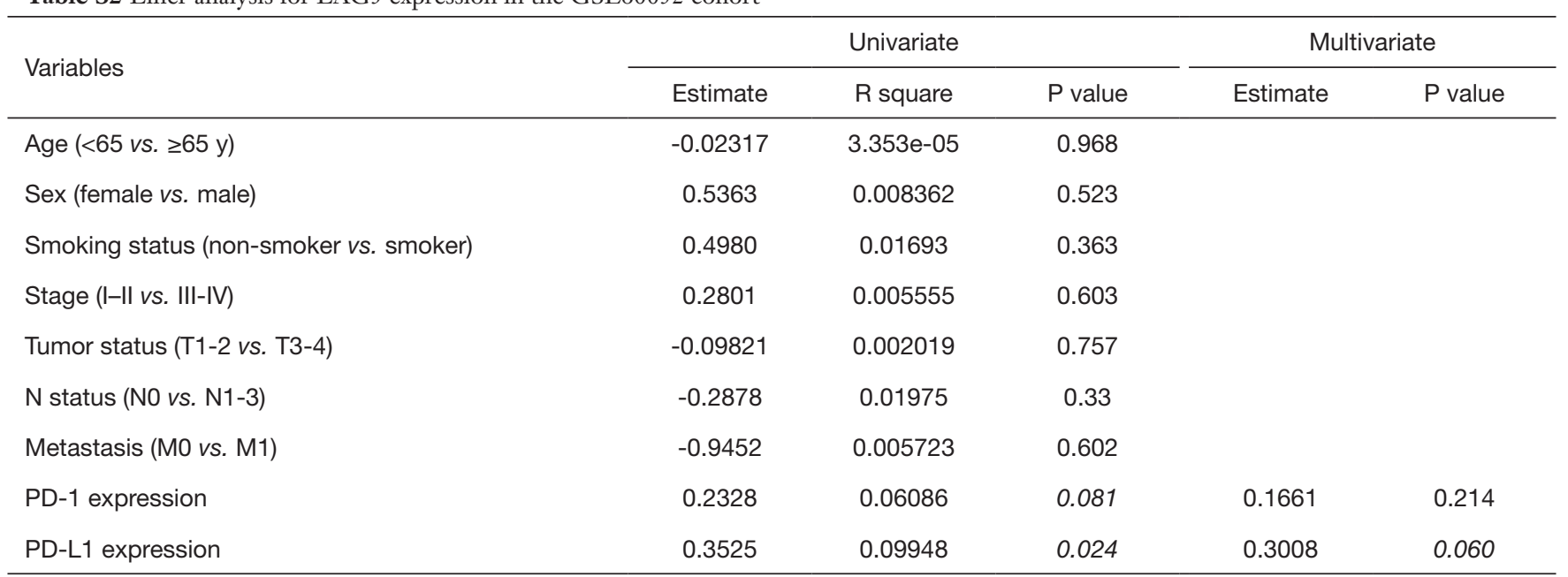

Data with $\mathrm{P}$ value less than 0.1 were marked with italics. LAG3, lymphocyte activation gene 3; PD-1, programmed death 1; PD-L1, programmed death ligand 1. 